\title{
Evaluation on a supersaturatable self-microemulsifying (s-smdds) formulation of biphenyl dimethyl dicarboxylate (BDD) in Vitro and in Vivo
}

\author{
Qingwei JIANG ${ }^{1}$, Tao WANG ${ }^{2 *}$ (D), Gao LI
}

\begin{abstract}
To enhance the dissolution and oral bioavailability of poorly water-soluble biphenyl dimethyl dicarboxylate (BDD), the supersaturatable self-microemulsifying drug delivery system (S-SMEDDS) was developed by adding a water-soluble polymer PVP to prevent precipitation of the drug and maintain a supersaturate state in vivo. Ternary phase diagrams were drawn to evaluate the microemulsification domain. The formulations were characterized by testing the physical stability of the drug, particle size and zeta potential. The pharmacokinetic study in beagle dogs was performed for the S-SMEDDS, SMEDDS formulation and the market drop pills. The optimized S-SMEDDS formulation consist of 35\% (w/w) Cremphor EL35, 33\% (w/w) Transcutol HP, $30 \%(\mathrm{w} / \mathrm{w})$ MCT and 2\% (w/w) PVPK30 of each excipient showed minimum mean droplet size $(37.71 \pm 0.87 \mathrm{~nm})$ and optimal drug release profile and better physical stability in water compared with the PVP absent SMEDDS. The in vivo studies showed that S-SMEDDS had significantly increased the $\mathrm{C}_{\max }$ and area under the plasma concentration-time curve $(A U C)(P<0.01)$. The S-SMEDDS formulation should be an effective oral dosage form for improving oral bioavailability of water-insoluble BDD.
\end{abstract}

Keywords: biphenyl dimethyl dicarboxylate; supersaturatable self-microemulsification; water-soluble polymer; accumulated dissolution; bioavailability;

Practical Application: This research can be applied to the oral absorption of plant active ingredients.

\section{Introduction}

Chronic hepatitis B is one of the serious diseases that threaten human health, the researchers were seeking for an effective therapy medicine ongoing in recent years. Biphenyl dimethyl dicarboxylate(BDD) was discovered in the chemical synthesis of schisandrin $C$ as a intermediate by scientist. It was applied to acute or chronic hepatitis therapy as liver-protection drug clinically for years (Cui et al., 2002; Kim et al., 2000). BDD is practically insoluble in water and poorly absorbed in vivo. The main dosage form of tablet and drop pill in the shipping product have a very low oral absorption which the absolute bioavailability are only $20 \%$ to $30 \%$.To overcome these problems, various formulation strategies were developed such as solid dispersion, solid lipid nanoparticles in the 1990s (Gu et al., 1990; Zhang et al., 2007), also including the application of self-microemulsifying drug delivery systems (SMEDDS) to improve oral bioavailability. The conventional SMEDDS are isotropic mixtures of an oil, surfactant, cosurfactant and drug. They form fine oil-in-water microemulsions less than $100 \mathrm{~nm}$ when introduced into aqueous media under mild agitation in $37^{\circ} \mathrm{C}$ (Pouton, 1997). The problem is that the drug must remain within the oil/water emulsion droplets following dilution of the SMEDDS formulation with the aqueous medium in the intestine. If the partition coefficient of the drug for the SMEDDS microemulsion particle is such that the solubility of the drug is exceeded in the aqueous phase, the drug could precipitate following dilution with water, and this could result in poor performance in vivo. A high level of the surfactant was added to conventional SMEDDS formulations in order to prevent precipitation of the drug following dilution with water in the gastrointestinal (GI) tract and, in some cases, the surfactants can lead to an increased incidence of GI side effects, especially for patients with chronic disease. Some researchers (Chen et al., 2010; Gao et al., 2003), attempted to put additives such as HPMC,MC,CMC-Na to SMEDDS whose aims were to inhibit the crystallization of drug which called supersaturatable self-microemulsifying drug delivery systems(S-SMEDDS). HPMC was used to generate a supersaturated state and crystallization of drug was inhibited in S-SMEDDS by Gao P (Gao \& Morozowich, 2006; Gao et al., 2009), Studies on the mechanism responsible for inhibiting crystallization of drugs in aqueous solutions containing HPMC suggests that the polymer chain may inhibit nucleation, as well as crystal growth by adsorption of the HPMC molecules onto the surface of the nuclei, or onto the surface of crystals (Miller et al., 2008; Gao et al., 2003; Kim \& Choi, 2002). In this study, polymer PVP was selected as precipitate inhibitor which showed better physical stability with less amount of surfactant compared with the SMEDDS. The optimized S-SMEDDS formulation was developed to improve the solubility and bioavailability of BDD and might bring higher efficacy and safety of oral BDD for a long term. 


\section{Materials and methods}

\subsection{Materials}

Biphenyl dimethyl dicarboxylate was provided by haixiang pharm.co.(Zhejiang, China), Medium Chain Triglyceride (MCT) was purchased from Lipoid(Germany), Polyoxyethylene castor oil (Cremophor EL-35) and PVPK90, PVPK30, PVPCf12 were obtained from Beijing Jingqiu Chemical Industry Co. Ltd. and Diethylene glycol monoethyl ether (Transcutol HP) was purchased from Gattefosse (France), respectively. All other chemicals and solvents were of reagent grade and used without further purification.

\subsection{Construction of ternary phase diagram}

The existence of self-microemulsifying fields were identified from ternary phase diagrams of systems containing oil-surfactantcosurfactant. A series of self-microemulsifying systems were prepared in each of the varying concentrations of oil MCT (0-43\%, w/w), surfactant Cremophor EL35 (35-100\%,w/w), cosurfactants Transcutol HP $(0-50 \%, w / w)$ and the amount of $\operatorname{BDD}(0.6 \%, \mathrm{w} / \mathrm{w})$ was added. The formulations $(2 \mathrm{~g})$ were introduced into $100 \mathrm{~mL}$ of $37^{\circ} \mathrm{C}$ water in a glass beaker and were mixed gently with a magnetic stir at $50 \mathrm{r} / \mathrm{m}$. The tendency to emulsify spontaneously and the droplets size of dispersed emulsions were observed and determined. Phase diagrams were constructed identifying the good self-microemulsifying region. All studies were repeated trice, with similar observations being made between repeats.

\subsection{Selection of the polymers}

The optimized SMEDDS formulations were prepared. Briefly, BDD was dissolved in oil and the appropriate amount of solvent (Transcutol HP) and surfactant (35\% Cremophor EL35, w/w) was added. Then, the components were mixed by gentle stirring at room temperature until the mixture solution turned to clear. Polymers such as hydroxypropyl methylcellulose (HPMC), methylcellulose (MC), sodium carboxymethylcellulose(CMC$\mathrm{Na}$ ), and polyvinyl pyrrolidone (PVP) was added into the mixture $(2 \%, w / w)$ and was stirring at $50{ }^{\circ} \mathrm{C}$ to obtain a uniform suspension or solution. All formulations were stored under room temperature before use. The apparent drug concentration-time profile and the duration of the supersaturated state in distilled water were determined as follows: Approximately $0.5 \mathrm{~g}$ of the SMEDDS and S-SMEDDS formulations (with types of polymers) placed in distilled water with volume of $100 \mathrm{~mL}$ maintained at $37^{\circ} \mathrm{C}$ and stirring at $50 \mathrm{r} / \mathrm{m}$. Then samples $(2 \mathrm{~mL})$ were taken at $1,2,4$ and $5 \mathrm{~h}$ and filtered through a $0.22 \mu \mathrm{m}$ filter. The initial fitrate was discarded and then assayed by a HPLC method for BDD as described in Ref. 2.4.

\subsection{HPLC assay for BDD}

The HPLC assay for samples from the in vitro experiments used a Zorbax ODS $4.6 \times 150 \mathrm{~mm}$ column, maintained at room temperature. Ultraviolet (UV) detection at $278 \mathrm{~nm}$ was employed. The mobile phase consisted of $0.2 \%$ triethylamine (adjust $\mathrm{pH}$ to 5.5 with $\mathrm{H}_{3} \mathrm{PO}_{4}$ ) and $50 \%$ acetonitrile at a flow rate of $1 \mathrm{~mL} / \mathrm{min}$,.

\subsection{Droplet size analysis and zeta potential}

S-SMEDDS $(0.5 \mathrm{~g})$ was diluted with $100 \mathrm{~mL}$ of water at $37^{\circ} \mathrm{C}$ in a glass beaker under stiring at $50 \mathrm{r} / \mathrm{m}$. The particle size and zeta potential of the resulting so-formed microemulsions was determined by Malvern-zs90 Electrophoretic light scattering particle size and zeta potential analyzer.

\subsection{Drug release studies}

Drug release studies from S-SMEDDS were performed using Pharmacopeia of China (CP) appendix XC, dissolution apparatus II with $500 \mathrm{~mL}$ of distilled water as medium at $37 \pm$ $0.5{ }^{\circ} \mathrm{C}$. The speed of the paddle was adjusted to $50 \mathrm{r} / \mathrm{m} .0 .5 \mathrm{~g}$ of the formulation encapsulated by soft gel (6mg of drug) was directly introduced into the medium and an aliquot $(5 \mathrm{~mL})$ of sample was collected at designated times $(5,10,15,20,30,45 \mathrm{~min})$ and analyzed for the content of BDD by HPLC as mentioned above. The commercial BDD drop pills were used as control. An equivalent volume $(5 \mathrm{~mL})$ of fresh dissolution medium was added to compensate for the loss due to sampling.

\subsection{In vivo study}

The pharmacokinetic study of BDD was conducted in beagle dogs fasted for $12 \mathrm{~h}(\mathrm{n}=6$, half male and female) in a crossover study design. The body weight of the beagle dogs ranged from 8 to $12 \mathrm{~kg}$; a fixed dose of BDD of $6 \mathrm{mg}$ from the reference formulation (BDD drop pill), SMEDDS or the S-SMEDDS formulation was used. One softgel containing the S-SMEDDS or SMEDDS formulation and two drop pills were administered orally to individual beagle dogs. In each case, $5 \mathrm{~mL}$ water was provided to each beagle dogs as flush liquid after administration. Dogs were allowed free access to water after dosing $4 \mathrm{~h}$. The washout time between each dosing phase of the study was one week. For each phase of the study, approximately $3 \mathrm{~mL}$ of blood was collected from each animal via the femoral vein into $5 \mathrm{~mL}$ tubes containing lithium heparin anticoagulant at $0.25 \mathrm{~h}, 0.5 \mathrm{~h}, 1 \mathrm{~h}$, $1.5 \mathrm{~h}, 2 \mathrm{~h}, 2.5 \mathrm{~h}, 3 \mathrm{~h}, 4 \mathrm{~h}, 5 \mathrm{~h}, 6 \mathrm{~h}, 8 \mathrm{~h}, 12 \mathrm{~h}$ and $24 \mathrm{~h}$. Blood tubes were centrifugation at $3750 \mathrm{r} / \mathrm{m}$ for $10 \mathrm{~min}$ to obtain plasma. Plasma $(1 \mathrm{~mL})$ was transferred to separate tubes and stored at $-20{ }^{\circ} \mathrm{C}$ until analysis. Our animal study was approved by the ethical committee of Academy of Military Medical Science of China. The date of approval was May fourth, 2011 and the number of identification was SCXK (jing) 2011-0003.

\subsection{Bioanalytical method for pharmacokinetic studies}

$100 \mu \mathrm{L}$ of internal standard solution was added into plasma samples $(1 \mathrm{~mL})$ and extracted using $5 \mathrm{~mL}$ volume of chloroform: ether $(3: 7, \mathrm{v} / \mathrm{v})$, then the samples were shaken for $5 \mathrm{~min}$ by vortex mixer and centrifuged at $3000 \mathrm{r} \bullet \mathrm{min}-1$ for $10 \mathrm{~min}$. The supernatant phase was separated and evaporated to dryness at $40^{\circ} \mathrm{C}$ under nitrogen. The dried residues were reconstituted with $200 \mu \mathrm{L}$ of mobile phase and samples were assayed by reversed-phase liquid chromatography on a Zorbax RX -C18 (5 $\mu \mathrm{m}, 4.6 \times 150 \mathrm{~mm})$ analytical column and UV detection at $278 \mathrm{~nm}$. The mobile phase was acetonitrile/MeOH/0.2\% triethylamine (H3PO4 adjust $\mathrm{pH}$ to5.5) (30:20:50, v/v/v) with a flow rate of $1 \mathrm{~mL} / \mathrm{min}$. A related molecule, diazepam, was used as the internal standard. 
The calibration curve was obtained by plotting the area ratios $\left(\mathrm{R}=\mathrm{A}_{\mathrm{BDD}} / \mathrm{A}_{\text {int }}\right)$ against the concentration of BDD. Good linearity was observed over a concentration range of $5 \sim 1000 \mu \mathrm{g} \bullet \mathrm{L}^{-1}$, with the correlation equation being $\mathrm{C}=1666.7 \mathrm{R}+1.8333(\mathrm{r}=0.9992$, $\mathrm{n}=3$ ). Limit of quantification was $3 \mu \mathrm{g} \bullet \mathrm{L}^{-1}$. At concentrations of 5,100 , and $500 \mu \mathrm{g} \cdot \mathrm{L}^{-1}$, extracted recoveries of BDD from plasma were $80.09 \%, 81.43 \%, 80.37 \%$; Intra-day precision was $8.10 \%$, $5.77 \%$, and $3.34 \%$; inter-day precision was $13.14 \%, 8.42 \%$, and $6.42 \%$; respectively. After storage for 1 month at $-20^{\circ} \mathrm{C}$ and freeze-thawing for three times, BDD was stable in plasma. Three concentrations of BDD quality control samples were prepared and assayed with every set of samples. The above results showed that the HPLC method was sensitive, precise and accurate for the determination of BDD in vivo.

\subsection{Statistical analysis}

Student's t-test or one-way analysis of variance (ANOVA) in SAS 9.2 was applied to analyze the significance of difference in vivo study. $\mathrm{P}$ values of $<0.01$ were indicative of statistically significant differences.

\section{Results}

\subsection{Construction of ternary phase diagrams}

A series of SMEDDS were prepared and their self-emulsifying properties were observed and ternary phase diagrams were constructed to identify the self-emulsifying regions which was shown in Figure 1.

\subsection{The in vitro supersaturation test}

As the S-SMEDDS formulation was designed to yield a supersaturated state, it was desired to have a meaningful test in vitro to assess the drug concentration sustained in the supersaturated state and estimate the degree of supersaturation as a function of time. In this work, the in vitro supersaturation test was designed to determine the apparent drug concentration-time profile

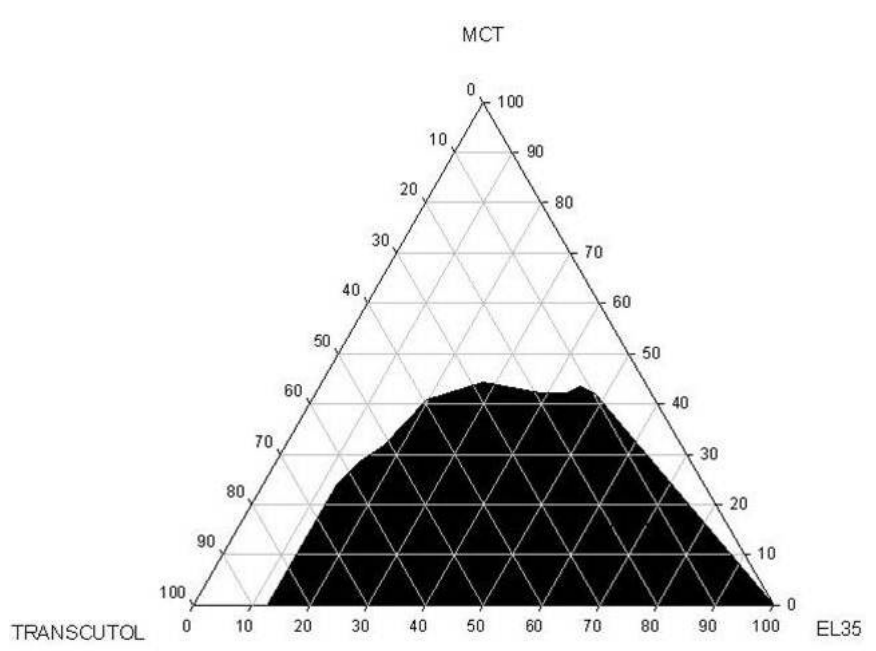

Figure 1. Ternary phase diagram. and the duration of the supersaturated state. For the purpose of screening formulations, a $0.22 \mu \mathrm{m}$ filter was used to process solution samples prior to HPLC analysis in order to limit the presence of precipitated drug particles of large size. A prototype SMEDDS formulation containing 35\% EL35 and five related S-SMEDDS formulations (e.g., the same composition with $2 \%$ HPMC, CMC-Na or PVP (k30, k90, 12pf) were evaluated by the supersaturation test. Due to the limited solubility of MC, the formulation with $2 \% \mathrm{MC}$ cannot form a clear solution, thus the $\mathrm{MC}$ was eliminated of the supersaturation test. The apparent $\mathrm{BDD}$ concentration-time profiles from the five formulations in water were shown in Figure 2. The S-SMEDDS formulation with 2\% PVPK30,PVPK90 showed a consistently higher apparent BDD concentration-time profile (plotted in Figure 2A) as compared to the prototype SMEDDS formulation without polymer and the S-SMEDDS formulation with 2\% PVP (12PF), HPMC or CMC-Na. The results suggested that polyvinyl pyrrolidone (PVP) K30 and K90 might inhibit BDD precipitation by sustained supersaturated state of the drug in water. Because of PVPK90 had a higher viscosity grades (PVPK90 > PVP K30) and longer dissolving process in formula, PVP K30 manifested the better candidate for the further comparative study. Further evaluations were directed to the S-SMEDDS formulations containing 35\% EL35 with 0, 0.5, 1, 2, and 5\% (w/w) PVP K30. This experiment was to evaluate the influence of the amount of PVP K30 upon the duration of the supersaturated state. As shown in Figure 2B, the stable apparent BDD concentration suggest that the presence of $0.5-2 \%$ PVP K30 appears effective sufficiently to prevent precipitation of the drug by generating and maintaining a supersaturate state. When the amount of PVP $\mathrm{K} 30$ was $5 \%$ in the S-SMEDDS formulation, the emulsification time was prolonged by causing a high viscosity with increased additives and resulted in shorter time of the supersaturated state sustained. The results suggested that the amount of polymer should be in a proper range.

\subsection{Determination of droplet size, zeta potential and emulsification time}

The rate of emulsification is an important index for the assessment of the efficiency of emulsification (Pouton, 1997), that is the SMEDDS should disperse completely and quickly when subjected to aqueous dilution under mild agitation. The prototype SMEDDS formulation containing 35\% EL35 and S-SMEDDS formulations with $0.5,1,2$, and $5 \%(w / w)$ PVPK30 containing 35\% EL35 were evaluated. Emulsification time study showed that the S-SMEDDS formulations with $0.5 \%(\mathrm{w} / \mathrm{w})$ PVPK30 emulsified efficiently as well with the prototype SMEDDS formulation, the other three S-SMEDDS formulations exhibited a little low rate of emulsification with the increased polymer amount of PVPK30. The results of droplet size analysis, zeta potential and emulsification time were shown in Table 1. Particle size study suggested that the presence of $0.5-2 \%(w / w)$ PVPK30 generated no significantly influence on the S-SMEDDS formulation, while the presence of 5\% (w/w) PVPK30 changed the droplet size of S-SMEDDS formulations. Among the tested formulations, zeta potentials kept the same level. 

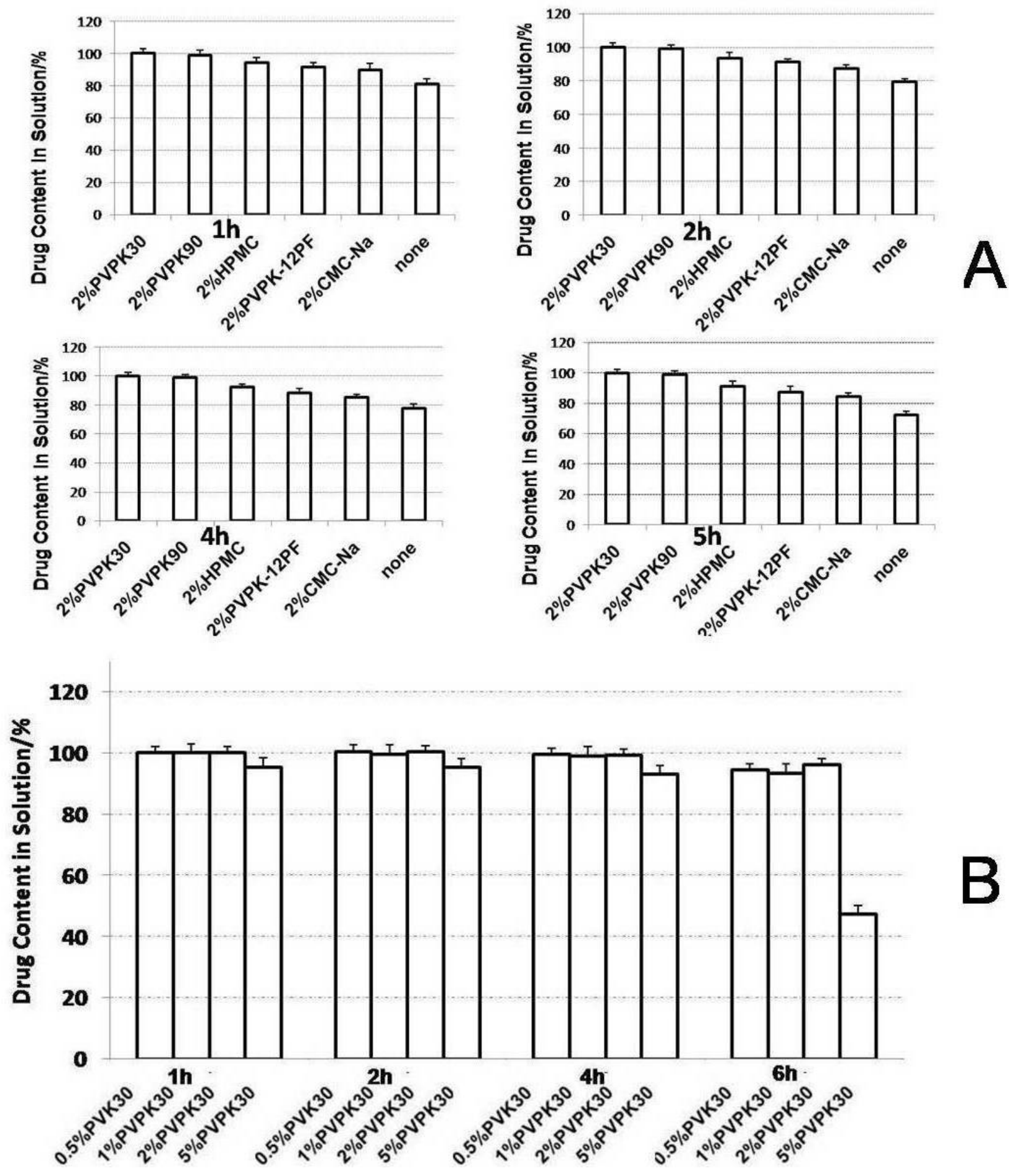

Figure 2. The BDD concentration-time profile of S-SMEDDS with different supersaturated additives.

Table 1. Particle size, zeta potential and emulsification time of S-SMEDDS formulations $(\mathrm{n}=3)$.

\begin{tabular}{cccc}
\hline Preparation & Emulsification time/s & Particle size/nm & Zeta potential/mV \\
\hline SMEDDS(35\%S) & $31 \pm 2$ & $36.86 \pm 0.65$ & $-7.31 \pm 0.35$ \\
SMEDDS(35\%S)+0.5\%PVP & $32 \pm 4$ & $37.12 \pm 0.46$ & $-7.91 \pm 0.54$ \\
SMEDDS(35\%S)+1\%PVP & $67 \pm 8$ & $37.24 \pm 0.67$ & $-7.87 \pm 0.83$ \\
SMEDDS(35\%S)+2\%PVP & $83 \pm 7$ & $37.71 \pm 0.87$ & $-7.21 \pm 0.44$ \\
SMEDDS(35\%S)+5\%PVP & $205 \pm 9$ & $51.68 \pm 1.04$ & $-7.54 \pm 0.69$ \\
\hline
\end{tabular}




\subsection{Drug release studies}

Drug release studies were performed with the S-SMEDDS and the reference drop pills. The results of the release profile of the two formulations in water were presented in Figure 3. BDD released less than $20 \%$ from the drop pill even at $45 \mathrm{~min}$ in water. Whereas, S-SMEDDS showed rapid dissolution, above $80 \%$ of BDD from S-SMEDDS released at $10 \mathrm{~min}$. In other words, S-SMEDDS could form clear and transparent solution quickly under the condition of dissolution. It was also suggest that S-SMEDDS enhanced dissolution of BDD significantly in vitro.

\subsection{In vivo study}

An oral bioavailability study was conducted in fasted beagle dogs ( $n=6$, crossover). The S-SMEDDS formulation was evaluated against the prototype SMEDDS formulation and the drop pill (as reference) which was used extensively in clinic. The plasma concentration-time profiles were plotted in Figure 4 . The maximum plasma concentration $\left(C_{\max }\right)$ and the corresponding peak time $\left(T_{\max }\right)$ were determined by the inspection of the individual drug plasma concentration-time. The pharmacokinetic parameters (e.g., the $A U C, T_{1 / 2 z}$ and $M R T$ ) of both formulations were calculated by DAS2.0 and summarized in Table 2. $A U C_{0-t}$ was calculated by the linear trapazoidol rule, $A U C_{0-\infty}$ was calculated as $A U C_{0-t}+C_{t} / K_{e}$. The statistic analysis

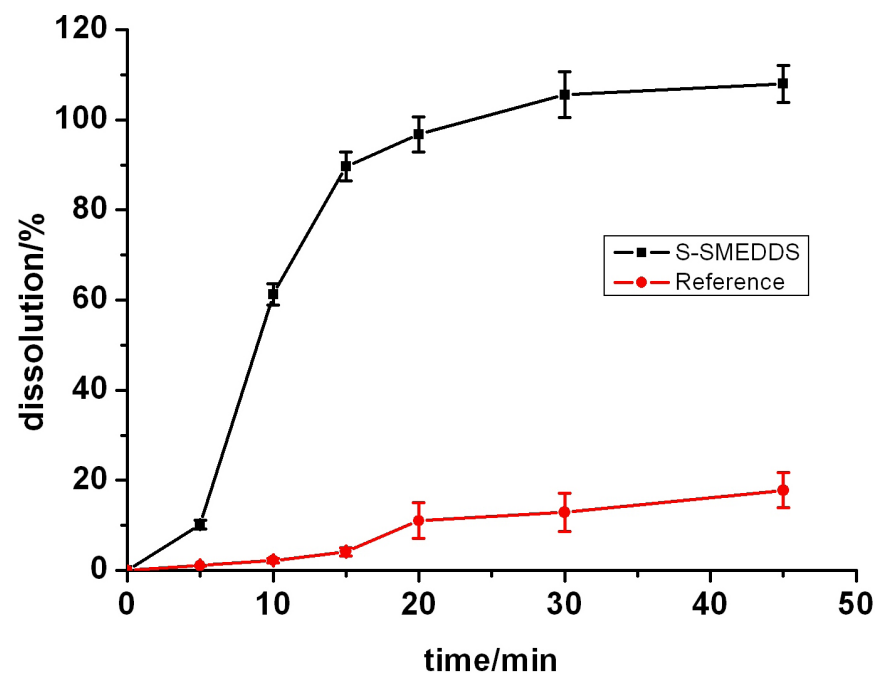

Figure 3. Accumulated dissolution curve of BDD preparations in vitro $(\mathrm{n}=6)$. of pharmacokinetic data by Paired $T$ test showed that $A U C_{0-t}$, $A U C_{0-\infty}$ and $C_{\max }$ had significant differences between S-SMEDDS and the reference, also S-SMEDDS and SMEDDS formulation. As expected, the reference formulation of BDD showed a low oral absorption. The S-SMEDDS gave significantly 2.47 -fold higher bioavailability $\left(A U C_{0-t}\right)$ than that of the reference $(P<$ 0.01). The SMEDDS formulation exhibited worse absorption in vivo compared to the S-SMEDDS formulation. This might due to the drug precipitate following dilution with water with the conventional SMEDDS without PVP, likewise the results of in vitro supersaturation test mentioned above in part 3.2 and lead to poor performance in vivo.

\section{Discussion}

S-SMEDDS can form a supersaturation state of free drug and retard the crystallization in vivo after oral administration when it emulsifying in the gastrointestinal (GI) fluid. In this study, various polymers were investigated for their ability to inhibit the crystallization of BDD in S-SMEDDS. The prevention of crystal formation by polymers was due to (a) prevention of crystal nucleation, (b) adsorption of the additives onto crystals and (c) formation of amorphous additive-drug co-precipitates (Jain \& Banga, 2010; Raghavan et al., 2003; Ziller \& Rupprecht, 1988). This study suggested that PVP (K30 and K90) presented in S-SMEDDS formulation generated a supersaturated state and

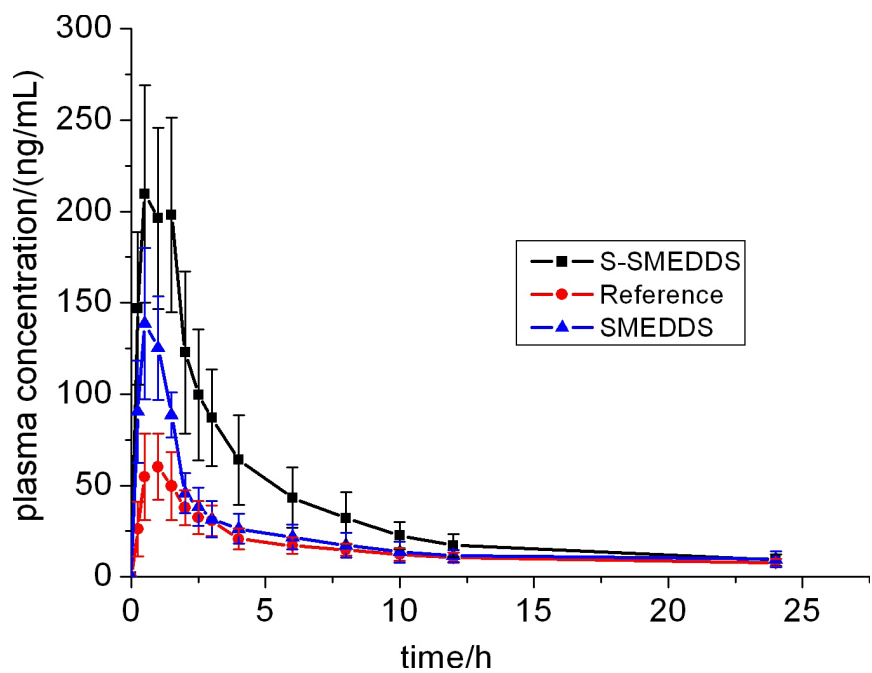

Figure 4. Plasma concentration-time profiles of BDD preparations in beagle dogs $(n=6)$.

Table 2. Pharmacokinetic parameters of BDD preparations in beagle dogs $(n=6)$.

\begin{tabular}{cccr}
\hline Parameters & Reference & SMEDDS & S-SMEDDS \\
\hline$A U C_{(0-\mathrm{t})} / \mu \mathrm{g} \cdot \mathrm{h} \cdot \mathrm{L}^{-1}$ & $378.94 \pm 71.43$ & $794.49 \pm 99.02^{*}$ & $935.91 \pm 185.09^{* *}$ \\
$A U C_{(0-\infty)} / \mu \mathrm{g} \cdot \mathrm{h} \cdot \mathrm{L}^{-1}$ & $473.94 \pm 80.07$ & $861.13 \pm 119.14^{*}$ & $1078.02 \pm 203.69^{* *}$ \\
$C_{\max } / \mu \mathrm{g} \cdot \mathrm{L}^{-1}$ & $60.13 \pm 18.13$ & $135.29 \pm 52.91^{*}$ & $209.61 \pm 119.49^{* \#}$ \\
$T_{\max } / \mathrm{h}$ & $1.25 \pm 0.59$ & $1.00 \pm 0.34$ & $1.00 \pm 0.49$ \\
$\mathrm{t}_{1 / 2 z} / \mathrm{h}$ & $11.20 \pm 6.36$ & $9.79 \pm 5.70$ & $10.64 \pm 8.26$ \\
$M R T_{(0-\mathrm{t})} / \mathrm{h}$ & $6.01 \pm 0.32$ & $5.32 \pm 0.75$ & $5.29 \pm 1.13$ \\
\hline
\end{tabular}

Note: ${ }^{*}$ compared to reference, $P<.0 .01$; \#compared to SMEDDS, $P<0.01$. 
showed a consistently higher apparent BDD concentration-time profile as compared to the prototype SMEDDS formulation containing the same content of surfactant without polymer. The favorable polymer PVPK30 was selected in the final S-SMEDDS formulation.

BDD is reported to possess poor solubility in water (only $3.2 \mathrm{mg} \cdot \mathrm{L}^{-1}$ ) that leading to its poor dissolution and poor delivery properties. According to the report, the solid dosage forms of BDD owe extremely poor in vivo absorption whose absolute AUC is only $20-30 \%$. In order to improve the soluble problem and absorption of BDD, this study utilized a S-SMEDDS formulation as carrier and achieved a significant improvement of dissolution in vitro and bioavailability in vivo. The S-SMEDDS improve the bioavailability may be attributed to the following factors: (a) larger surface area provided by the fine emulsion droplets, (b) improved diffusion of the fine emulsion droplets, (c) increased mucosal permeability due to surfactants and (d) improved lymphatic absorption due to the oil (Balakrishnan et al., 2009).

\section{Conclusion}

The S-SMEDDS of BDD was formulated which a water-soluble polymer PVP was added to prevent precipitation of the drug by generating and maintaining a supersaturate state. A significant improvement of dissolution in vitro and bioavailability in vivo were achieved. The S-SMEDDS formulation should be an effective oral dosage form for improving oral bioavailability of water-insoluble BDD.

\section{Acknowledgments}

This work was supported by The National Natural Science Foundation of China (Grant No. 81102390).

\section{References}

Balakrishnan, P., Lee, B. J., Oh, D. H., Kim, J. O., Lee, Y. I., Kim, D. D., Jee, J. P., Lee, Y. B., Woo, J. S., Yong, C. S., \& Choi, H. G. (2009). Enhanced oral bioavailability of Coenzyme Q10 by self-emulsifying drug delivery systems. International Journal of Pharmaceutics, 374(1-2), 66-72. http://dx.doi.org/10.1016/j.ijpharm.2009.03.008. PMid:19446761.

Chen, Y., Du, R., Zeng, X. F., Shi, Q. Z., \& Liu, H. (2010). Effect of supersaturated promoter on the dissolution of self-emulsifying drug delivery system of docetaxel. Journal of Chinese Pharmaceutical Sciences, 45(6), 444-449.

Cui, S.N., Wang, Z. H., Li, L. X., Zu, Y. H., \& Wang, M. M. (2002). Antiviral efficacy of oral Bifendate for a long term in treatment of patients with chronic Hepatitis B. Chinese journal of integrated traditional and Western Medicine. 12(3), 144-146.
Gao, P., \& Morozowich, W. (2006). Development of supersaturatable self-emulsifying drug delivery system formulations for improving the oral absorption of poorly soluble drugs. Expert Opinion on Drug Delivery, 3(1), 97-110. http://dx.doi.org/110. 10.1517/17425247.3.1.97.

Gao, P., Akrami, A., Alvarez, F., Hu, J., Li, L., Ma, C., \& Surapaneni, S. (2009). Characterization and optimization of AMG 517 supersaturatable self-emulsifying drug delivery system (S-SEDDS) for improved oral absorption. Journal of Pharmaceutical Sciences, 98(2), 516-528. http:// dx.doi.org/10.1002/jps.21451. PMid:18543293.

Gao, P., Rush, B. D., Pfund, W. P., Huang, T., Bauer, J. M., Morozowich, W., Kuo, M. S., \& Hageman, M. J. (2003). Development of a supersaturable SEDDS (S-SEDDS) formulation of paclitaxel with improved oral bioavailability. Journal of Pharmaceutical Sciences, 92(12), 2386-2398. http://dx.doi.org/10.1002/jps.10511. PMid:14603484.

Gu, S. J., Wang, X. L., Gao, W. W., Qiao, P. X., Wang, A. G., Qiang, Z. Y., \& Song, Z. Y. (1990). Bioavailability studies on the preparations of biphenyl dimethyl dicarboxylate. Yao Xue Xue Bao, 25(3), 215 219. PMid:2239337.

Jain, P., \& Banga, A. K. (2010). Inhibition of crystallization in drugin-adhesive-type transdermal patches. International Journal of Pharmaceutics, 394(1-2), 68-74. http://dx.doi.org/10.1016/j. ijpharm.2010.04.042. PMid:20438819.

Kim, J. H., \& Choi, H. K. (2002). Effect of additives on the crystallization and the permeation of ketoprofen from adhesive matrix. International Journal of Pharmaceutics, 236(1-2), 81-85. http://dx.doi.org/10.1016/ S0378-5173(02)00017-0. PMid:11891072.

Kim, J. H., Mun, Y. J., Chun, H. J., Jeon, K. S., Kim, Y. O., \& Woo, W. H. (2000). Effect of biphenyl dimethyl dicarboxylate on the humoral immunosuppression by ethanol. International Journal of Immunopharmacology, 22(11), 905-913. http://dx.doi.org/10.1016/ S0192-0561(00)00053-9. PMid:11090699.

Miller, D. A., Dinunzio, J. C., Yang, W., McGinity, J. W., \& Williams, R. O. 3rd (2008). Targeted intestinal delivery of supersaturated itraconazole for improved oral absorption. Pharmaceutical Research, 25(6), 1450-1459. http://dx.doi.org/10.1007/s11095-008-9543-1. PMid:18288449.

Pouton, C. W. (1997). Formulation of self-emulsifying drug delivery systems. Advanced Drug Delivery Reviews, 25(1), 47-58. http:// dx.doi.org/10.1016/S0169-409X(96)00490-5.

Raghavan, S. L., Schuessel, K., Davis, A., \& Hadgraft, J. (2003). Formation and stabilisation of triclosan colloidal suspensions using supersaturated systems. International Journal of Pharmaceutics, 261(1-2), 153-158. http://dx.doi.org/10.1016/S0378-5173(03)00299-0. PMid:12878404.

Zhang, H., Zhan, X. A., \& Cheng, B. (2007). Preparation of Bifendateloaded solid lipid nanoparticles. J Guangdong College of Pharmacy, 23(6), 37-40.

Ziller, K. H., \& Rupprecht, H. (1988). Conteol of crystal growth in drug suspensions: 1) Design of a Conteol unit and application to acetaminophen suspensions). Drug Development and Industrial Pharmacy, 14(15-17), 2341-2370. http://dx.doi.org/10.3109/03639048809152019. 